This item was submitted to Loughborough's Research Repository by the author.

Items in Figshare are protected by copyright, with all rights reserved, unless otherwise indicated.

\title{
Reducing the transmission and reception powers in the AODV
}

PLEASE CITE THE PUBLISHED VERSION

PUBLISHER

(C) IEEE

VERSION

VoR (Version of Record)

LICENCE

CC BY-NC-ND 4.0

REPOSITORY RECORD

Abusaimeh, Hesham, and Shuang-Hua Yang. 2019. "Reducing the Transmission and Reception Powers in the AODV”. figshare. https://hdl.handle.net/2134/5116. 
This item was submitted to Loughborough's Institutional Repository (https://dspace.lboro.ac.uk/) by the author and is made available under the following Creative Commons Licence conditions.

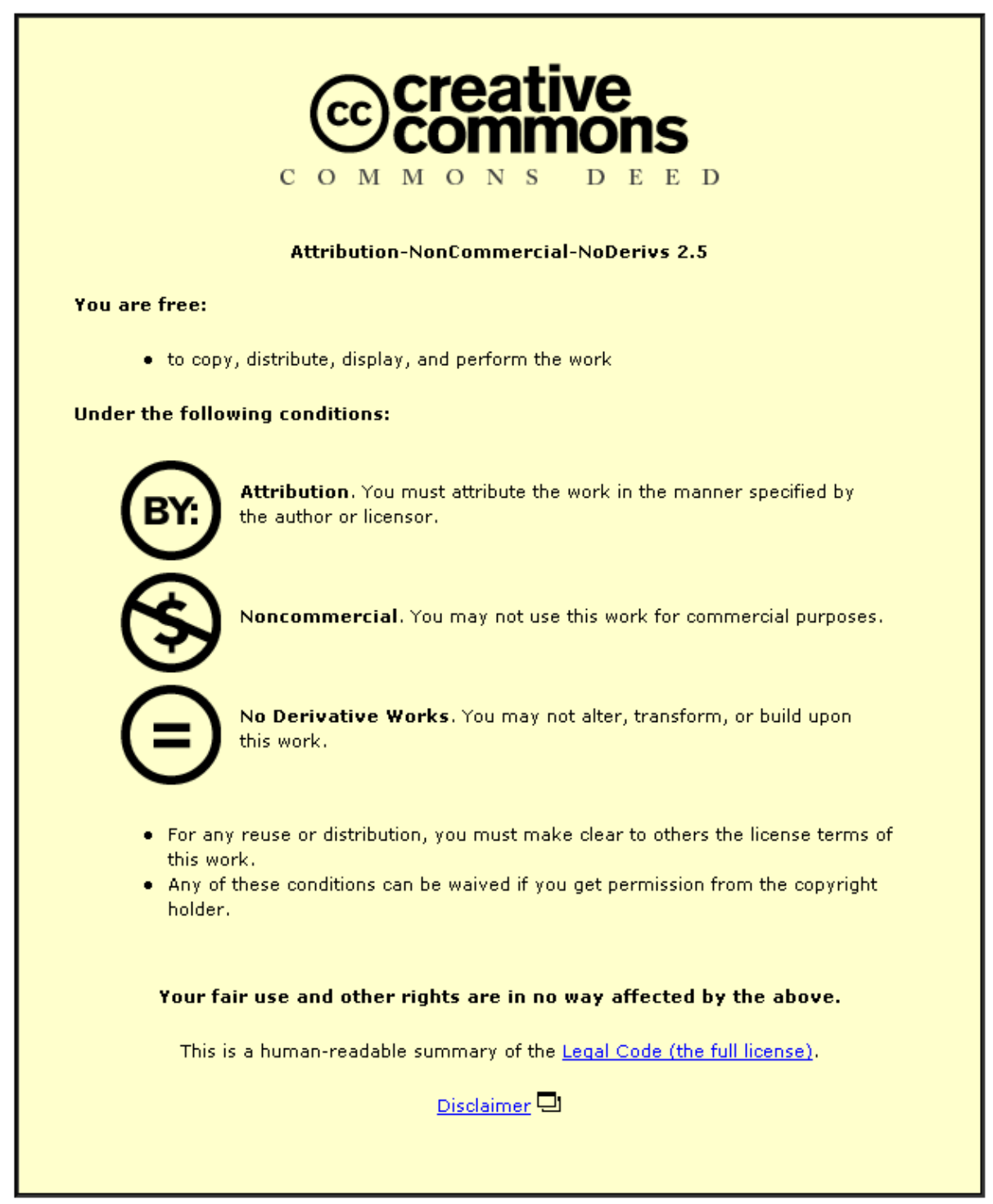

For the full text of this licence, please go to: http://creativecommons.org/licenses/by-nc-nd/2.5/ 


\title{
Reducing the Transmission and Reception Powers in the AODV
}

\author{
Hesham Abusaimeh, Shuang-Hua Yang (SMIEEE) \\ Computer Science Department \\ Loughborough University \\ Loughborough, UK \\ H.S.Abusaimeh@lboro.ac.uk, S.H.Yang@lboro.ac.uk
}

\begin{abstract}
The transmission power and the reception power are the main source of energy consumption in wireless sensor nodes. Therefore, reducing the amount of consumed power through the transmission and reception processes in the routing protocol will lead to an increase in the lifetime of the wireless sensor network and distribute the consumed power among the wireless sensor nodes. This paper proposes a mechanism to reduce the transmission and reception power for the frequently used nodes. This proposed techniques and a hybrid technique with the power consumption distribution technique in [1] have been compared to the original Ad hoc On-Demand Distance Vector (AODV) routing protocol. Simulation results based on NS-2 showed that the hybrid technique and the proposed mechanism have better performance than the original AODV routing protocol. The new mechanism and the hybrid technique have increased the lifetime of the wireless sensor network by around $40 \%$, and $70 \%$ respectively.
\end{abstract}

Keywords; Energy Consumption; AODV; Network Lifetime; Routing; WSN; Transmission Power; Reception Power.

\section{INTRODUCTION}

W ireless Sensor Networks (WSNs) have appeared as a consequence of advanced research on real time, low cost, long battery life, and low data rate wireless applications. WSNs have been designed to serve many types of applications requiring limited power, reliable data transfer, short range communication, and reasonably low cost such as industrial monitoring and control, home automation and security, and automotive sensing applications [2]. A WSN is a set of sensors that communicate with each other to form the sensor field. A WSN consists of a large numbers of nodes, which have the ability to communicate wirelessly, to perform limited computation, and to sense their environment [3]. Specific functions can be obtained by cooperation among these nodes [4].

Wireless sensors accomplish their functions in the network by having the ability to send and receive signals between each other. Each wireless sensor contains a battery as a power source, as well as a small memory unit, and computational unite capable of performing some simple operations when required. Wireless sensors can be made from different materials such as metals, polymers and plastics, ceramics and glass, biological materials, semiconductors etc. Additionally it can be made from a combination of two or more of these materials [5].

These sensors can be used in many real time applications in the world such as monitoring environmental changes, controlling security needs for houses and spaces, estimating the traffic flows, monitoring military systems and many other applications in environmental, health, safety, and tracking systems [3].

In order to perform successfully in these applications, the wireless sensors may often apply to be located in inaccessible areas and difficult conditions. Therefore, they need to be stable in design and able to reliably transfer data between the wireless sensors in such conditions for the maximum period of time. In addition, they need to consume as little power as possible while providing a long active working life because of the limited power source available in each sensor. In many applications, it is very difficult or even impossible to change the batteries of these sensors. For example, they can be installed in critical locations such as the bottom of the ocean, or inside tornados [2]. Consequently, designing efficient wireless sensor networks is much more difficult than any other compatible computer network devices. This is due to both the limitation in the resources such as the power source, computation unit, and memory size, and also the harsh environments of the applications.

There are many routing protocols for dealing with data transfer over wireless sensor nodes. Some of these protocols are concerned with the Quality of Service (QoS). Others are concerned with energy consumption. AODV protocol is one of the most efficient routing protocols in terms of shortest path and power consumption. The latest technology in WSN, the Zigbee standard, uses this protocol in its routing layers [6]. However, this routing protocol does not consider the energy level of the intermediate nodes in the routing process.

Many researchers are working on increasing the network lifetime of the WSNs based-on three possible solutions to the problem of power consumption by the wireless sensor nodes. They are adding extra batteries to the wireless sensor node, creating a technique to distribute the power between the nodes, and making the nodes responsible for finding their own power source [7]. This paper has produced a technique to distribute the consumption power among the wireless sensor nodes by controlling the transmission and the reception powers.

In the data transmission process from a source node to a destination node, all the sensor nodes use the same communication standard and routing protocol. Therefore, the amount of energy consumed for transmitting one packet to the destination node is the same and fixed for each sensor node involved in the routing process [4]. Consequently, reducing the amounts of the reception and 
transmission powers from one node to another, will save energy, and increase the lifetime of these nodes.

The objective of this paper is to establish a model of reducing the transmission and the reception powers during the routing process and to investigate its efficiency on the WSNs, and on distributing the consumption power among the wireless sensor nodes. This technique distributes the power consumption among the nodes, and can achieve better performance than the original AODV routing protocol in terms of reducing the rate at which the energy is consumed, while increasing the lifetime of the wireless sensor nodes and the lifetime of the WSN.

The rest of this paper is organised as follows. In section II, the proposed new technique is presented. The implementation of this new mechanism over the basic AODV is explained in section III. In section IV, the simulation environment, parameters, and results are discussed for the wireless sensor network. Section V contains the conclusion.

\section{PROPOSED MECHANISM}

In this section, we introduce a new mechanism to balance the consumption energy in the network by preventing critical nodes from consuming all their energy before other nodes in the network do. These critical nodes spend most of their energy in the transmission and reception processes rather than the standby or any other status.

The main idea of the proposed technique is that the node, that has high energy, will cover the most area, while the node, that has less energy, will cover the least area. This happens by decreasing the amounts of the transmission and the reception powers for the nodes that reduce their energy very quickly, as a result of forwarding a large number of packets in the route between the source and the destination. Therefore, these nodes will cover less area than the other nodes in the network. However, the minimum amount of the transmission and reception powers preserved should be enough to transmit and receive a packet for the distance between the node and its neighbours.

Our idea is based on Cheng et al. who proposed a dynamic power management system in [8], to balance the consumption of power among the nodes in the network based on reducing the transmission power by the following formula:

$$
P t=\text { PtInit } * \frac{\operatorname{Pr} e s}{P f}
$$

Where $P t$ is the transmission power, PtInit is the power spent on the initial transmission, Pres is the residual power, and $P f$ is the full power.

However, this proposed model didn't cover the reception power as well. Therefore, reducing the transmission power is not sufficient to balance the consumption power in the WSN. In addition, Cheng et al. did not consider the distance between the nodes, and the reception power in reducing the amount of the transmission power. In addition, their dynamic power management system could reduce the transmission power to a value less than that required power for delivering a packet to a neighbour node, which would lead to stopping the node from taking part in the communication process in the WSN, where it actually has some power to continue to work and serve the network. In our model, the nodes will keep serving the network until loosing all their power.

In our model, each node can adjust its transmission power and reception power individually based on its remaining and initial energy. We reduced the transmission power and the reception power based on the following mathematical formulas:

$$
\begin{aligned}
& P t=\text { PtInit } * \frac{\operatorname{Re} m E n g}{\text { InitEng }} \\
& \operatorname{Pr}=\operatorname{Pr} \text { Init } * \frac{\operatorname{Re} m E n g}{\text { InitEng }}
\end{aligned}
$$

Where $\operatorname{Pr}$ is the reception power, PrInit is the initial reception power, RemEng is the remaining energy of the node, and InitEng is the initial full energy of the node.

We calculate the remaining energy of the node based on our energy model in [1]. However, this proposed technique attempts to reduce the transmission power and the reception power until reaching the minimum power, which is required to transmit the packet between two nodes in a certain distance. The relationship between the transmission power, the received power and the distance can be found from the following formula [9]:

$$
\operatorname{Pr}=\frac{P t^{*} G t^{*} G r^{*} h t^{2 *} h r^{2}}{d^{4} * L}
$$

Where $G t$ is the antenna gain for the transmitter, $G r$ the antenna gain for the receiver, $h t$ is the height of the transmitter antenna, $h r$ is the height of the receiver antenna, $L$ is the system loss, and $d$ is the transmission distance.

\section{IMPLEMENTATION}

In this section, we describe two implementations. The first one is for the previous technique of reducing the transmission and reception power of the low energy nodes over the basic AODV routing protocol. The second implementation is for a hybrid technique of this technique using the technique in [1] for distributing the consumption power and reducing the consumption power average for the wireless sensor nodes.

In order to implement this technique, some steps have been added to the basic AODV routing protocol. These steps are as follows:

Step 1: Any wireless sensor node that needs to send a message; it has to check its routing table and look for a path to the destination node. Therefore, if the route is available in the routing table, it will forward the message to the next node. Otherwise, the message will be saved in a queue, and the source node will send the RREQ packet to its neighbours to initiate the discovery process.

Step 2: We have reduced the transmission and reception powers of the node involved in the forward 
process based on equations (2) and (3). However, the minimum value of these powers should be enough to forward the data packet over the minimum distance to reach the next neighbour in the route.

Step 3: In order to merge this technique with the consumption power distribution technique in [1] to produce a hybrid technique, we have implemented a procedure before forwarding the message to the next hop. This procedure checks the energy consumption speed of the next hop. If the energy consumption speed is high, then the procedure will turn off the next hop for a certain period of time. After that, the current route will be removed from the routing table, which will lead the source node to initiate the discovery process again and find a new path to the destination node.

These two implementations are explained in the following flow charts:

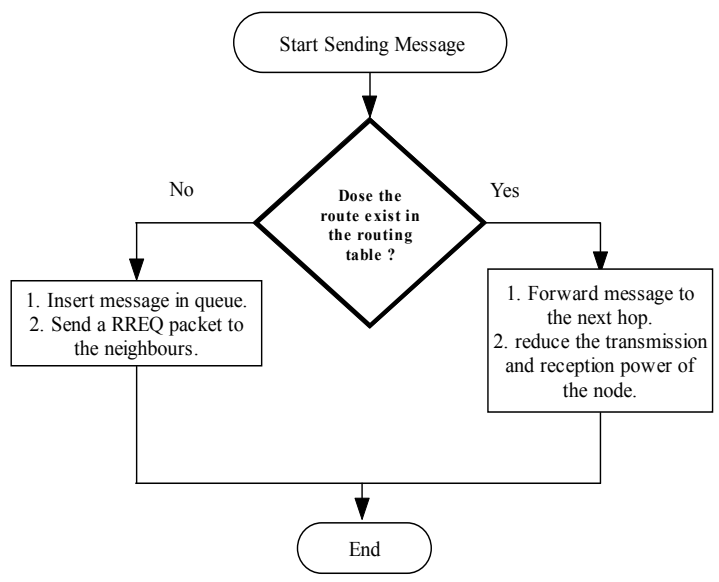

Figure 1: Flow Chart to implement the new technique over the basic AODV.

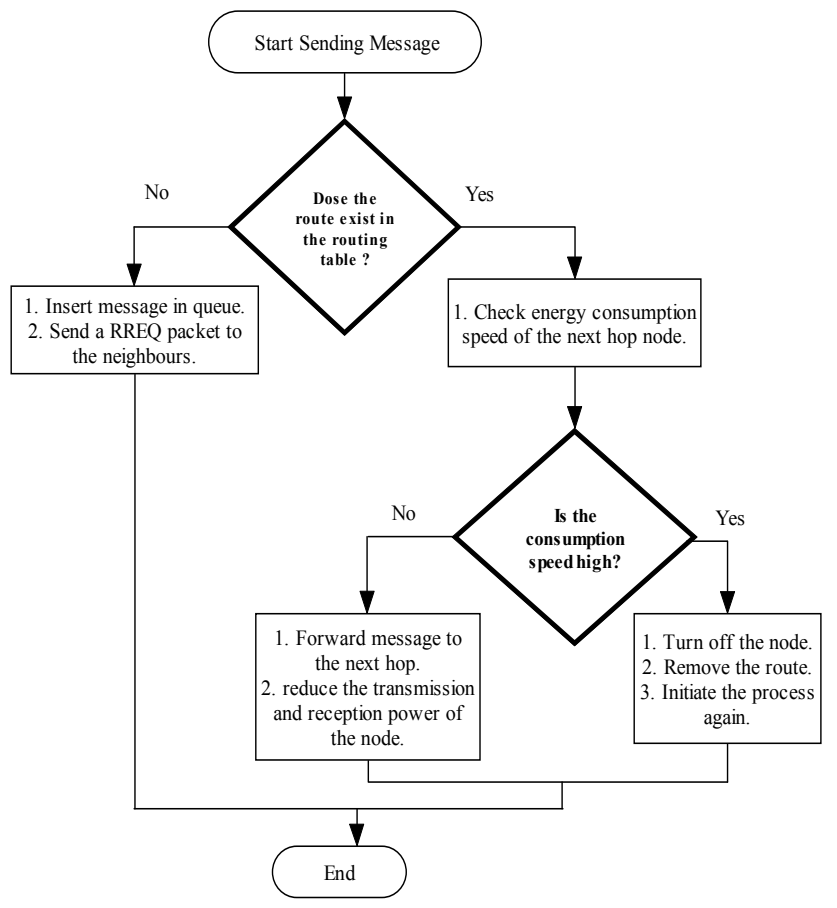

Figure 2: Flow Chart to implement the hybrid technique.

\section{Simulation MOdEL AND ReSUlts}

This section describes the simulation environment, and shows the results of the simulation of the proposed technique of reducing the transmission and the reception powers of the wireless sensor nodes, and the simulation results of the hybrid technique between this new mechanism and the power consumption distribution technique. Both of them are based on the basic AODV routing protocol. This simulation is accomplished using the Network Simulator NS-2 version 2.29.2 [10]. The NS2 simulation used Ad hoc On Demand Vector (AODV) as a routing protocol to discover and maintain the routes between the source and the destination nodes. Moreover, this simulation used IEEE802.15.4 as a Medium Access Control (MAC) protocol. IEEE802.15.4 is a standard definition for the Physical layer (PHY) and the MAC layer of the low Rate Wireless Personal Area Networks (LRWPANs) which support the communication of simple devices such as wireless sensors [11].

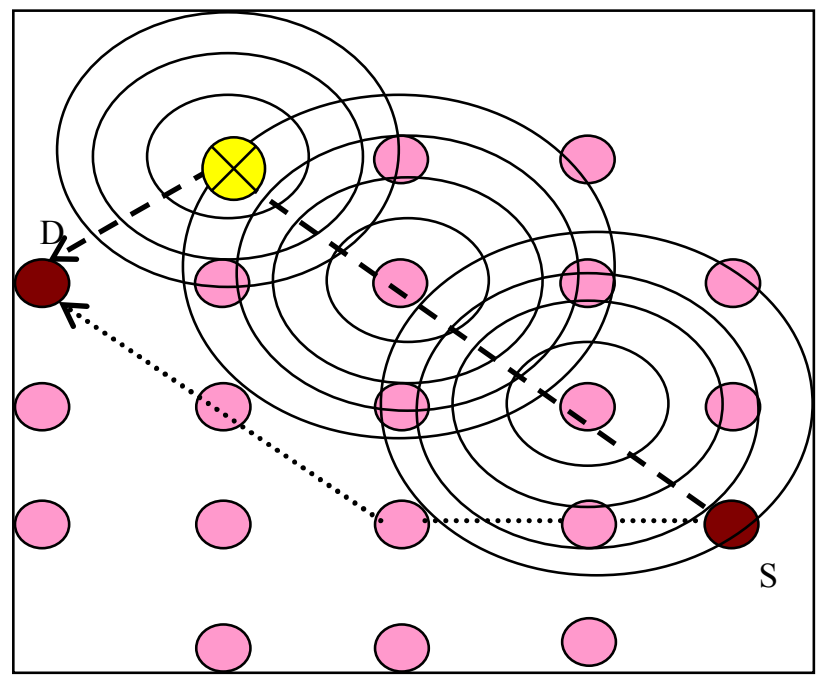

Figure 3: Proposed model diagram showing one node with less transmission and reception power.

The simulation model consists of 21 wireless sensor nodes as shown in Figure 3 in an area of size 80x80 with the TwoRayGround radio model. These sensor nodes are distributed systematically. The distance between any two neighbour nodes is 16 meters. The range of the wireless signal transmission initially is 30 meters. A traffic flow is generated between a source and a destination node. The traffic flow type is Constant Bit Rate (CBR) traffic connection with packet size 1000 bytes. This CBR starts after 0.5 second of the simulation start time and redundant each 15 millisecond. This means the rate of the traffic flow is equal to 4000 bytes CBR packets per second. Each simulation runs for 500 seconds.

This simulation uses the NS-2 energy model, which represents the energy level of the wireless sensor nodes. The energy model includes three initial values: the initial energy (initialEnergy ), the transmission power of each packet (rxPower_), and the reception power of each packet (txPower_). The output of this model is the total remaining energy, packets transmission energy, and the reception energy of each node. This NS2 energy model 
decreases the node energy, when the node transmits and receives packets by applying the following equations [9].

$$
\begin{aligned}
& \text { Node_Eng }=\text { Node_Eng }-\left(P t^{*} \text { txTimes }\right) \\
& \text { Node_Eng }=\text { Node_Eng }-\left(\mathrm{Pr}^{*} r x \text { Times }\right)
\end{aligned}
$$

Where Node Eng is the node energy and initially is equal to the initial energy of the node, txTimes is the number of the transmitted packets, and rxTimes is the number of the received packets.

In this simulation, the initial full energy is equal to 4.0 joules, the transmission and the reception powers are each equal to 1.0 watt. These simulation values and other simulation parameters are shown in the following Table 1.

Table 1 Simulation Parameters Values

\begin{tabular}{|l|c|}
\hline \multicolumn{1}{|c|}{ Parameter Name } & Parameter Value \\
\hline InitEng & 4.0 Joules \\
\hline $\mathrm{Pr}$ & $1.0 \mathrm{Watt}$ \\
\hline $\mathrm{Pt}$ & $1.0 \mathrm{Watt}$ \\
\hline Packet Size & 1000 Bytes \\
\hline Packet Interval & 15 Milliseconds \\
\hline Simulation Time & 500 Seconds \\
\hline Number of Nodes & 21 \\
\hline Antenna Type & OmniAntenna \\
\hline Radio Model & TwoRayGround \\
\hline Grid Size & $80 \mathrm{X} 80$ \\
\hline Distance between neighbours & $16 \mathrm{~m}$ \\
\hline Routing Protocol & AODV \\
\hline MAC Protocol & MAC/802_15_4 \\
\hline CPThresh_ & 10.0 \\
\hline CSThresh_ & $2.13643 * \mathrm{e}^{-7}$ \\
\hline RXThresh_ & $2.13643 * \mathrm{e}^{-7}$ \\
\hline L_ & 1.0 \\
\hline Gt & 1.0 \\
\hline Gr & 1.0 \\
\hline Ht & $1.5 \mathrm{~m}$ \\
\hline Hr & $1.5 \mathrm{~m}$ \\
\hline
\end{tabular}

The simulation is based on the two-ray ground reflection model. The transmitter antenna gain, and the receiver antenna gain are $1(G t=G r=1)$. The transmitter antenna height is the same as that of the receiver antenna height and equal to $1.5(h t=h r=1.5)$. The system loss of the antenna is equal to $1(L=1)$. Therefore, we can simplify equation (4) to the following simplified form:

$$
\operatorname{Pr}=\frac{5.0625^{*} P t}{d^{4}}
$$

As shown in Figure 3, the crossed wireless sensor nodes in the dashed route has less transmission and reception powers than the other nodes in the same route. In addition, the dotted route is used because the approach in [1] detect that one node in the dashed route has lost its power quickly, so it turned that node off for a small period of time, which caused the source node (S) to find another route in order to reach the destination node (D). By repeating these processes of reducing the transmission and the reception powers, and distributing the role of routing data among the wireless sensor nodes of the WSN as it's implemented over the AODV routing protocol, we have achieved better performance.

In this simulation, performance indexes are compared for three protocols: the basic AODV, the new technique, and the hybrid technique. They are the speed of the energy consumption of the wireless sensor nodes, the lifetime of the nodes, and the lifetime of the wireless sensor network. The performance evaluation of these protocols has been carried out under the same traffic source, the same network loads, the same parameters, and the same simulation environments.

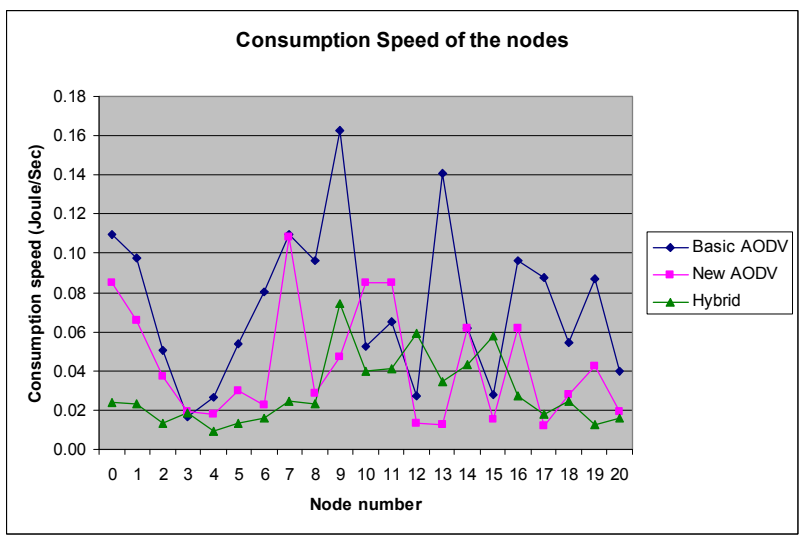

Figure 4: Energy consumption speed of the wireless sensor nodes of the three techniques.

Figure 4 shows the simulation result for the energy consumption speed of each node over the three protocols. The new technique has reduced the energy consumption speed for most of the nodes to less than the basic AODV routing protocol. However, the hybrid technique has decreased the energy consumption speed for most of the nodes to less than the basic AODV and the new technique. While, the average of the energy consumption speed of the wireless sensor nodes in the basic AODV was around 0.074 joule per second, the new technique has reduced the average to reach around 0.043 joule per second. However, the hybrid technique has decreased the average of the energy consumption speed to 0.029 joule per second.

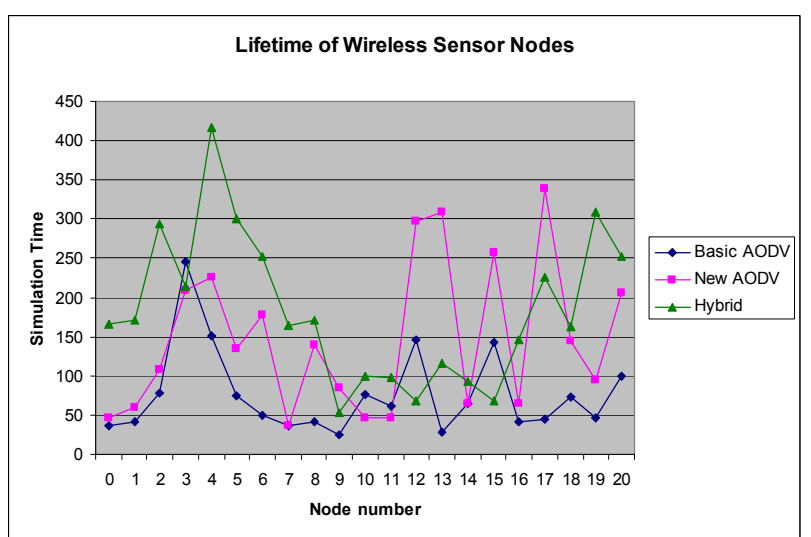

Figure 5: Lifetime of the wireless sensor nodes of three protocols. 
Consequently, the lifetime of the wireless sensor nodes has been increased by both the new technique and the hybrid technique comparing with $n$ the original lifetime of the nodes using the basic AODV. The new technique has raised the average lifetime of the wireless sensor node to reach 147 seconds. The hybrid technique, however, has increased the average lifetime to reach 183 seconds. Where, the original average lifetime of the wireless sensor nodes was around 76.5 seconds as shown in Figure 5.

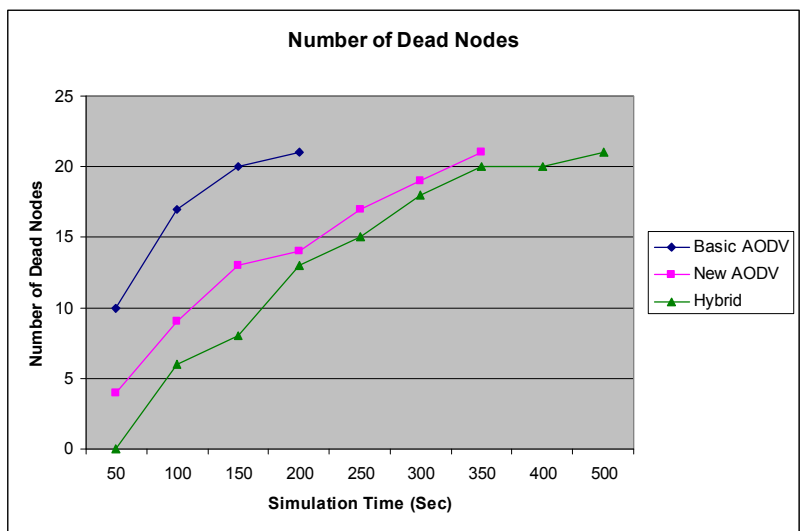

Figure 6: Lifetime of the wireless sensor network over the three techniques.

If we can assume that the lifetime of the WSN is equal to the maximum lifetime of the wireless sensor nodes, and all nodes can achieve the same role and functionality if they stay alive, the lifetime of the wireless sensor node can be calculated by applying the following equation [1]:

$$
\text { LifeTime }=\frac{\text { InitEng }}{\text { ConsSpeed }}
$$

Where, Consspeed is the consumption speed of the wireless sensor node. The following equation calculates the consumption speed [1].

$$
\text { ConsSpeed }=\frac{\text { InitEng }- \text { Re } m \text { Eng }}{\text { TimePeriod }}
$$

Where, TimePeriod is the running time of the wireless sensor node.

The lifetime of the WSN in this simulation was around 240 seconds based on the basic AODV routing protocol. Figure 6 clearly shows that the new technique has increased the lifetime of the WSN to 338.5 seconds. The hybrid technique has almost doubled the lifetime of the WSN to be around 416 seconds.

It is obvious from all the previous simulation results that the new technique has achieved better performance than the basic AODV routing protocol. However, the Hybrid technique has achieved the best performance in terms of reducing the average energy consumption speed of the wireless sensor node, increasing the average lifetime of the wireless sensor nodes, and increasing the lifetime of the whole wireless sensor network.

\section{CONCLUSION}

AODV routing protocol is a widely used in wireless sensor networks, which is based on both the IEEE802.15.4 and ZigBee standards of the WSN. However, this protocol does not take into consideration of the changes in the battery energy level of the wireless sensor nodes through the communication or the routing processes. This may lead to critical problems in routing data, and may limit the network lifetime. Energy consumption is a significant concern to many researches concerned with WSN.

In this paper, we have proposed a new technique to decrease the transmission and the reception powers of the wireless sensor nodes based on the remaining energy level to the minimum power level which is still sufficient to transmit a single packet across a fixed distance between the node and its neighbours. Subsequently, we have merged this new technique with the technique proposed in [1] to reduce the consumption among the wireless sensor nodes by distributing the role of routing among the wireless sensor nodes, and requiring most of them to participate in the communication process. This proposed new technique together with the hybrid technique aim to increase the lifetime of the whole network, and to increase the number of nodes, which will remain active for a maximum period of time.

The performance evaluation in terms of the network lifetime, power consumption of the nodes, and the nodes lifetime, was conducted using the NS-2 simulator. We have compared the original AODV routing protocol with the new technique and the hybrid technique under the same simulation conditions and parameters values. Results showed that the new proposed technique has improved the performance in terms of increasing the total network lifetime, and decreasing the energy consumption speed of each wireless sensor node in the WSNs by around $40 \%$.

Based on the simulation results, the hybrid approach has achieved better performance than the original AODV routing protocol, as it has increased the lifetime of the whole WSN by around $70 \%$ in the WSN.

\section{ACKNOWLEDGMENT}

Hesham Abusaimeh would like to acknowledge the financial support of Applied Science University (ASU) Faculty of Computer Science and Information Technology in The Hashemite Kingdom of Jordan for his PhD study.

\section{REFERENCES}

[1] Abusaimeh, H., and Yang, S., 2008, "Balancing the Power Consumption Speed in Flat and Hierarchical WSN", International Journal of Automation and computing, Vol. 5, No. 4, pp 366-375, October 2008.

[2] Akyidiz, I.F., Su, W., Sankarasubramaniam, Y., and Cyirci E., 2002, Wireless Sensor Network: a survey, IEEE Communication Magazine,Vol. 38, No. 4, March 2002.

[3] Romer, K., Kastin, O., and Mattern, F., Middleware Challenges for Wireless Sensor Networks, Vol. 6, No. 4, pp 59-61, 2002.

[4] Shorey, R., Ananda A., Chan M.C., and Ooi W. T.., Mobile, Wireless, and Sensor Networks, IEEE press \& John Wiley \& Sons., 2006.

[5] Eren, H., Wireless Sensor and Instruments, Taylor \& Francis, 2006.

[6] Ergen S.C., 2004, Zigbee/IEEE802.15.4 Summary, September 2004. 
[7] Roundy, S.,Wright, P.K., and Rabaey, J.M., 2004, "Energy Scavenging For Wireless Sensor Network", Kluwer Academic Publishers, 2004.

[8] Cheng, L., Bourgeois, A.G., and Yu, B. H., 2005, "Power Management in Wireless Ad Hoc Networks using AODV", the $6^{\text {th }}$ international conference on software engineering, artificial intelligence, networking and parallel/ distributed computing, IEEE, 2005.

[9] Fall K., and Varadhan K., 2007, The ns Manual (formerly ns Notes and Documentation), $19^{\text {th }}$ Jan 2007.
[10] USC Information Sciences Institute, Marina del Rey, CA. Network Simulator - NS2, http://www.isi.edu/nsnam/ns

[11] IEEE Std 802.15.4-2003, 2003, IEEE Standard for Information technology -Telecommunications and information exchange between systems - Local and metropolitan area networks Specific requirements - Part 15.4: Wireless Medium Access Control (MAC) and Physical Layer (PHY), Specifications for LowRate Wireless Personal Area Networks (LR-WPANs), 2003 